\title{
Philosophiques
}

Ludwig Feuerbach, Éthique : l'Eudémonisme, 1867-1869 suivi de

L'homme est ce qu'il mange, 1862. Traduction d'Anne-Marie

Pin, préface de Roger Bruyeron, Paris, Hermann, 2012, 140 p.

\section{Emmanuel Chaput}

Volume 40, numéro 1, printemps 2013

URI : https://id.erudit.org/iderudit/1018386ar

DOI : https://doi.org/10.7202/1018386ar

Aller au sommaire du numéro

Éditeur(s)

Société de philosophie du Québec

ISSN

0316-2923 (imprimé)

1492-1391 (numérique)

Découvrir la revue

Citer ce compte rendu

Chaput, E. (2013). Compte rendu de [Ludwig Feuerbach, Éthique :

l'Eudémonisme, 1867-1869 suivi de L'homme est ce qu'il mange, 1862.

Traduction d'Anne-Marie Pin, préface de Roger Bruyeron, Paris, Hermann,

2012, 140 p.] Philosophiques, 40(1), 234-238. https://doi.org/10.7202/1018386ar d'utilisation que vous pouvez consulter en ligne.

https://apropos.erudit.org/fr/usagers/politique-dutilisation/ 
pas dans le volume. Sur le fond, si la question du pragmatisme est centrale aux yeux des interprètes non métaphysiques, on peut se demander si les arguments du processualisme de Whitehead ne pourraient pas contribuer à ces recherches, de même que le rapprochement tout récent entre la philosophie hégélienne et les modèles contemporains en physique théorique. La première n'est mentionnée qu'une fois (dans la note i9 de l'article de Renault) et la seconde ne l'est pas du tout. Les critiques du lien entre pensée hégélienne et pragmatisme sont nombreuses en langue anglaise, mais peu apparentes ici $^{8}$. Le problème méthodologique qui peut, au début, créer un effet de circularité (pour situer Hegel par rapport à la métaphysique, il faut définir celle-ci; pour définir la métaphysique, il faut situer la pensée hégélienne par rapport à elle), se dissipe rapidement devant le très vaste éventail de réponses proposées. On ne peut reprocher au volume de manquer d'une unité à laquelle il ne prétend pas et qu'il se défend bien de rechercher. Le tout est impressionnant et témoigne d'une pensée vive. On peut très justement le comparer en importance au collectif dirigé par Alasdair MacIntyre, Hegel: A Collection of Critical Essays, en I97I.

JEANNE ALLARD

Université de Montréal

\section{Ludwig Feuerbach, Éthique: l'Eudémonisme, 1867-1869 suivi de L'homme est ce qu'il mange, 1862. Traduction d'Anne-Marie Pin, préface de Roger Bruyeron, Paris, Hermann, 2012, 140 p.}

Essentiellement connu par le lectorat francophone pour son œuvre maîtresse L'essence du christianisme (I84I), Ludwig Feuerbach (I804-I872) eut une influence certaine, bien que trop souvent ignorée, sur tout un pan de la philosophie post-hégélienne. Louis Althusser écrivait en effet: "On constatera avec quelle étonnante profondeur Feuerbach annonce et devance, à un titre ou à un autre, par contraste ou parenté, Marx, Nietzsche, Freud, Husserl, certains thèmes de Heidegger, Barth et la théologie récente ${ }^{9}$.» Cette perspec-

8. Entre autres, Houlgate, S. "Phenomenology and de re interpretation: a critique of Brandom's reading of Hegel»; Stern, R. "Hegel and Pragmatism» dans A Companion to Hegel, Wiley-Blackwell, Oxford, 20I I, p. 556-575 et Hegelian Metaphysics, Oxford University Press, Oxford, 20I 2, spécialement p. 48-50; Redding, P. «The Analytic Neo-Hegelianism of John McDowell and Robert Brandom» dans A Companion to Hegel, Wiley-Blackwell, Oxford, 20I I, p. 576-593 et "Analytic Philosophy and the Return of Hegelian Thought", Cambridge University Press, Cambridge, 2010 [2007]; Macdonald, I. "Nature and Spirit in Hegel's Anthropology: Some Idealist Themes in Hegel's Pragmatism» dans Laval théologique et philosophique, 63: I, février 2007, p. 4I-50. Il faut souligner, à la défense des auteurs, que ces critiques sont, pour la plupart, antérieures à la parution du présent volume, mais postérieures à la tenue du colloque de 2009 .

9. Cité dans la préface de Roger Bruyeron, p. 32. 
tive ouvre les horizons de l'interprétation de l'œuvre de Feuerbach trop souvent circonscrit à n'être qu'un auteur pré-marxiste, important dans la genèse de la pensée du jeune Marx, mais dont la critique religieuse et métaphysique est rapidement appelée à être dépassée par la critique pratique de Marx. Comme le rappelait Engels dans son ouvrage de I 888: "nous fûmes tous momentanément des "feuerbachiens" " ${ }^{10}$. Momentanément, car Feuerbach est souvent vu, surtout dans les études marxiennes, comme un moyen terme entre l'idéalisme allemand et le matérialisme pratique de Marx.

Certains, tel Alexis Philonenko ${ }^{11}$, ont voulu arracher la pensée de Feuerbach au monopole de l'herméneutique marxienne (ou althussérienne) afin de restituer sa légitimité à la fois comme penseur critique anti-théologien et comme historien de la philosophie. Néanmoins, dans tous les cas, les études françaises portant sur Feuerbach se concentrèrent toujours principalement sur son œuvre de jeunesse. Autant l'ouvrage de Philonenko ne va pas au-delà de I84I, autant la traduction d'Althusser des Manifestes philosophiques - de même que la traduction de Yannis Constantinidès parue aux éditions Mille et une nuits - restent concentrées sur les textes des années 40. C'est en quelque sorte à cette lacune que l'ouvrage paru chez Hermann vient répondre. Avec sa traduction de textes des années 6o, Anne-Marie Pin fournit au lectorat francophone un accès au Feuerbach de la maturité.

L'intérêt de ces textes est d'abord de voir à quel point l'entreprise critique de Feuerbach n'est complète qu'à la lumière d'une éthique, pendant pratique et positif à la dimension critique de sa pensée. À la base de l'éthique feuerbachienne on retrouve un instinct universel, principe métaphysique Feuerbach dirait naturel - qui conditionne tout agir: l'instinct du bonheur. La volonté n'agit pas pour elle-même, mais d'abord en réaction au mal et à la misère, en vue du bonheur. Feuerbach prétend ainsi trouver dans la volonté de bonheur le motif initial de toute entreprise humaine, qu'elle soit individuelle, communautaire, universelle, révolutionnaire ou conservatrice. Évidemment, parmi les différentes formes - égoïste, altruiste, individualiste, etc. — que prend l'instinct de bonheur, certaines sont plus conformes à la nature que d'autres. Ainsi, pour Feuerbach, le respect de soi est avant tout un respect de l'autre: "Respect assurément de toi-même — l'Autre seul représente le devoir ${ }^{12}$.» L'égoïsme authentiquement éthique prend également en considération l'alter ego. C'est à ce titre, quand la misère d'autrui m'est aussi intolérable que mon propre malheur, que la situation ouvrière décrite par Marx dans le Capital (cité par Feuerbach) devient si scandaleuse. L'agir moral n'est possible que sur une base matérielle qui n'est pas la misère la plus crasse

10. F. Engels, Feuerbach et la fin de la philosophie classique allemande, Paris, Éditions sociales, I966, p. 23.

11. A. Philonenko, La jeunesse de Feuerbach I828-i84I (2 vol.), Paris, Vrin, I990.

12. L. Feuerbach, Éthique: l'Eudémonisme, I867-1869 suivi de L'homme est ce qu'il mange, I 862, Paris, Hermann, 20I 2, p. 99. 
condamnant l'ouvrier à ne s'exprimer que dans ses fonctions animales les plus basses, et qui, par son activité proprement humaine, le travail, ne s'exprime plus du tout, mais s'aliène. Dans cette perspective, l'éthique feuerbachienne aspire à une morale tenant compte du désir de bonheur de tous en s'assurant des conditions matérielles nécessaires à cette aspiration. En cela, Feuerbach s'inscrit dans l'entreprise que Marcuse associe à la théorie marxiste: "L'idée de Raison se trouve désormais éclipsée par l'idée de bonheur ${ }^{13}$.»

L'Éthique s'oppose ainsi à l'idéalisme pour qui l'activité de la volonté est circonscrite à la pensée, alors qu'au contraire l'activité réelle est justement la négation de la pensée, en tant qu'elle veut la dépasser dans une transformation effective de la réalité. La pratique réelle motivée par l'instinct du bonheur n'appelle pas simplement à une correction dans la configuration de notre pensée, mais à un changement véritable de la situation de nos contemporains les plus misérables. Le devoir moral, contrairement à ce qu'en ont pensé les prêtres — religieux ou philosophiques — de ce monde, n'a d'autre contenu que l'eudémonisme, l'aspiration au bonheur, que ce soit le sien ou celui d'autrui. Feuerbach est ainsi plus proche de l'eudémonisme grec d'un Aristote que de ces contemporains allemands incapables de saisir dans leur spéculation philosophique le rôle fondamental du bonheur réel comme motif de l'activité.

Toutefois, lucide, Feuerbach reconnaît que l'instinct de bonheur ne fait pas toujours le bonheur de l'homme. Au contraire, en premier lieu et le plus souvent, l'instinct de bonheur prend la forme de son contraire, le malheur, le mal, la misère. Toujours, chez Feuerbach, c'est le positif qui engendre sa propre négation, et non l'inverse, le malheur est l'espoir déçu d'un bonheur. Par certains aspects, cette approche du désir est plus proche des analyses de l'Anti-Edipe de Deleuze et Guattari que de Freud. Il n'y a qu'un instinct, celui du désir, qui peut en s'exprimant finir par s'opprimer. On retrouve ici le même schéma que dans l'aliénation religieuse, où l'imagination de l'homme, en exprimant ses désirs d'infini dans un au-delà transcendant, finit par constituer la principale entrave à sa réalisation dans l'ici-bas.

Force est donc de constater qu'à travers ses textes de la maturité, Feuerbach poursuit son entreprise philosophique initiée au cours des années 30-40 d'une critique de la théologie et de la philosophie spéculative. Néanmoins cette entreprise critique s'appuie de plus en plus sur une dimension naturaliste qui fait parfois sourire, voire sursauter le lecteur contemporain tant ce naturalisme d'époque peut paraître aujourd'hui malvenu. Paradoxalement, malgré ses intuitions fortes, l'auteur des Principes de la philosophie de l'avenir (I 843) n'a peut-être pas été en mesure de dépasser les préjugés de son époque. C'est ainsi que son matérialisme éthique qui pose la nécessité d'une base matérielle comme condition de possibilité à tout agir moral devient problématique lorsqu'il s'agit de comparer la civilisation européenne aux

13. H. Marcuse, Raison et révolution, Paris, Éditions de Minuit, I968, p. 34I. 
cultures dites primitives. En effet, dans la mesure où les sens jouent un rôle essentiel dans la philosophie feuerbachienne, l'hygiène de vie, en tant qu'elle aiguise la sensibilité et développe ainsi l'horizon des expériences possibles, permet une meilleure intelligence du monde et des moyens en vue du bonheur. Là encore, Feuerbach s'appuie sur une position essentiellement aristotélicienne selon laquelle c'est à partir de l'intériorisation de l'expérience sensible que procède, par voie d'abstraction, l'intelligence ${ }^{14}$. Cela implique cependant qu'une sensibilité plus faible permet une expérience moindre du monde. Dès lors, l'éthique feuerbachienne prend un tournant hygiéniste de bien mauvais aloi: la brute sauvage n'a ainsi qu'une expérience partielle du monde, moindre que l'homme raffiné, sensible à l'hygiène, à la propreté et aux parfums fins qui s'offrent à lui. Bien entendu, cela n'exclut pas que le sauvage puisse parfaitement se satisfaire de son expérience. Son bonheur est cependant, pour Feuerbach, bien campé dans sa position évolutionniste, celui de l'ignorant auquel il faut préférer le bonheur du sage.

Le second texte du recueil Le mystère du sacrifice ou L'homme est ce $q u$ 'il mange - dont une traduction d'Anne-Marie Pin avait déjà paru en 2008 chez Stalker — prend pour point de départ le célèbre aphorisme feuerbachien "Der Mensch ist was der isst.» Feuerbach s'en sert comme prétexte pour développer à nouveau ses thèses sur le rapport entre l'homme et la religion. Comme il le remarque justement, par le sacrifice, l'homme entre en communion avec les dieux, mais cette communion trouve son point de départ à la table, dans la communion du repas, lieu de la socialité: "Les sacrifices sont des repas pris en commun par les dieux et les hommes ${ }^{15}$."

Toutefois si Dieu, comme l'homme, est ce qu'il mange, sa nourriture doit être le reflet de son essence, comme la nourriture de l'homme est également reflet de l'homme: «Dieu est ce qu'il mange; il mange l'ambroisie [...] un mets immortel [...] l'homme par contre mange du pain, les fruits de la terre $[. .$.$] des nourritures mortelles { }^{16}$.» La créature terrestre se nourrit des fruits de la terre alors que le créateur céleste se nourrit des fumées, des odeurs des bêtes immolées en sacrifice à son autel. On retrouve ici la manifestation de l'aliénation religieuse, explicitée à partir du sacrifice: l'homme pose ses propres pratiques, sa propre essence - celle d'un être vivant devant se nourrir — dans l'au-delà divin sans toutefois y poser ses limites. Ainsi, la satisfaction gastronomique prend chez l'homme un tour tragique lorsqu'il s'exprime comme manque, pour le misérable tiraillé par la faim. On retrouve alors dans la nutrition des dieux une nouvelle figure du mouvement que Feuerbach exposait dans son Essence du christianisme. Les dieux ne connaissent que le plaisir de la chair sans jamais connaître la faim.

14. Aristote, De l'âme, 43 2a, I-I4.

15. L. Feuerbach, op.cit., p. II 5 .

16. Ibid., p.IO4 


\section{8 • Philosophiques / Printemps 2013}

Bien qu'elles n'aient pas la fougue des textes de jeunesse ( I 830-I 845), les nouvelles traductions d'Anne-Marie Pin offrent au lecteur francophone l'occasion d'apprécier les développements de la pensée de Feuerbach qui s'inscrit de plus en plus dans le primat de la réalité sensible et naturelle, tout en opposant à la morale du devoir ou de l'utilité — autrement dit la morale chrétienne ou bourgeoise - la nécessité de tirer des leçons de la sagesse des Anciens.

EMMANUEL CHAPUT

Université de Montréal

\section{Paul Thagard, The Cognitive Science of Science, MIT Press,} Cambridge (MA)/Londres, 2012, $365 \mathrm{p}$.

Il y a quelques mois paraissait dans la revue Science un article décrivant Spaun, un cerveau artificiel comportant 2,5 millions de neurones, capable de réaliser huit tâches cognitives, dont une version simplifiée d'un test d'intelligence ${ }^{17}$. Cette prouesse des neurosciences théoriques, héritières du connexionnisme d'antan, constitue une bonne occasion de se demander si celles-ci ont quelque chose à apporter à la philosophie. The Cognitive Science of Science de Paul Thagard pourrait être la réponse à cette question, et c'est dans cet esprit que nous présenterons son ouvrage. Thagard est l'un de ceux qui (comme les Churchland) ont poursuivi le projet d'une philosophie neurocomputationnelle des sciences et, dans ce livre où il collabore, entre autres, avec certains des membres du laboratoire derrière Spaun, il apporte à l'étude des sciences la nouvelle révolution neurale en sciences cognitives: l'application des neurosciences théoriques aux fonctions de haut niveau du cerveau - dans ce cas-ci, celles qui sont essentielles à la pratique scientifique. Malgré une certaine volonté de multidisciplinarité, les neurosciences théoriques occupent en effet la place centrale dans la version des sciences cognitives de la science proposée par Thagard. Il a développé une approche de la science par simulations neuronales, laquelle permet à la fois de décrire les mécanismes cognitifs en jeu dans la pratique scientifique et, par le fait même, grâce à une conception instrumentale de la rationalité scientifique, de mieux cerner les normes de la science - deux tâches qui ont traditionnellement occupé les philosophes.

Pour comprendre ces modèles, il faut d'abord dire un mot sur les neurosciences théoriques qui les animent. Cela donnera en même temps une idée des progrès faits par celles-ci et de leur capacité à réanimer les vieux débats philosophiques sur les modèles neuronaux, car de la résolution de ces débats dépend en partie la valeur des modèles de la science de Thagard. Il

17. C. Eliasmith, T. C. Stewart, X. Choo, T. Bekolay, T. DeWolf, Y. Tang, D. Rasmussen (20I2). "A Large-Scale Model of the Functioning Brain », Science, 338(6I I I), I 202-I 205. 